\title{
FLAMINGO NoE Project Management of the Future Internet
}

\author{
Sebastian Seeber \\ University of Munich, Germany \\ sebastian.seeber@unibw.de
}

\begin{abstract}
The FLAMINGO project will strongly integrate the research of leading European research groups in the area of network and service management, strengthen the European and worldwide research in this area, and bridge the gap between scientific research and industrial application.
\end{abstract}

\section{Introduction}

FLAMINGO [1] has several objectives, such as to lead the scientific network and service management community, to organize conferences and workshops, develop open source software, establish joint labs, jointly supervise Ph.D. students, develop educational and training material, interact with academia and industry as well as to contribute to (IETF and IRTF) standardization. There are three major and federating challenges which will be investigated: At first the Network and service monitoring, based on flow-based techniques, enabling scalable monitoring systems to share collected data and feed the knowledge plane and decision algorithms of the Future Internet (FI). Next is automated configuration and repair, based on self-* features and frameworks, enabling billions of devices and managed objects to manage themselves in a fully distributed and autonomic way. Last the economic, legal, and regulative constraints, which do border management systems and operational solutions of the FI.

\section{Key Issues and Problems}

The FLAMINGO project is focused around three key research activities (see Figure 1 below). Before any management decision can be taken, it is essential to first monitor the managed objects (networks, services, traffic, devices etc.). The output of the monitoring process is than used to automatically configure and repair the managed objects. Monitoring as well as the automated configuration and repair should be performed within the boundaries of the economic, legal and regulative constraints.

Monitoring: To make informed network and service management decisions, it is essential to have a thorough understanding of the traffic. The FLAMINGO consortium will focus on new flow-analysis techniques, propose novel distributed techniques to capture and analyse management data, and develop new query languages to detect flow patterns. These novel approaches will be validated for the purpose of security management and privacy awareness.

A. Galis and A. Gavras (Eds.): FIA 2013, LNCS 7858, pp. 344-345, 2013.

(C) The Author(s). This article is published with open access at link.springer.com 


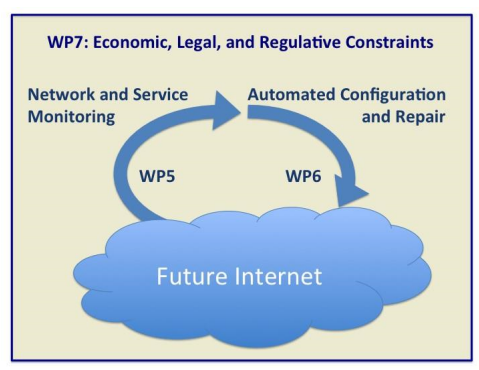

Fig. 1. Interaction between the different Workpackages

Automated Configuration and Repair: Due to the billions of objects that need to be managed, and the resulting scalability problems, it is necessary to rethink existing management approaches. A centralized and hierarchical approach can no longer address these challenges adequately. What we need is automated configuration and repair of managed objects, such that these objects react to their environment in an adaptive way, according to certain rules. These novel concepts will be investigated and validated by the application in content-aware networking, optical networks, cloudbased services, personal and wireless sensor networks.

Economic, Legal, and Regulative Constraints: The Future Internet will see management decisions to be taken and optimized, based not only on technological, but also on commercial deployment and economic viability concerns. Therefore a crossdisciplinary approach is needed, taking into account a) costawareness, b) incentives for service provisioning, c) fulfilment schemes, d) business policies, and e) legal/regulative aspects. These aspects are complemented by legal and regulative constraints, which have to be evaluated to ensure that contracts concluded will be legally valid and provider-dependent cost models as well as accounting models are legally compliant with regulations.

\section{$3 \quad$ Expected Results}

The impact of the FLAMINGO Network of Excellence will be a strong European position in the field of network and service management. To achieve this, FLAMINGO creates training material to facilitate researchers within industry to keep up with the latest developments in this field. The project develops network management course material and makes such material available to universities worldwide. It will develop technologies for future generations of European high speed networks, in particular scalable monitoring techniques and network components that operate autonomically. FLAMINGO will contribute to standardization, in particular within the IETF to the evolution of the NETCONF and YANG standards, as well as several other working groups. FLAMINGO also organizes prestandardization in this area within the IRTF Network Management Research Group.

Open Access. This article is distributed under the terms of the Creative Commons Attribution Noncommercial License which permits any noncommercial use, distribution, and reproduction in any medium, provided the original author(s) and source are credited.

\section{Reference}

1. FLAMINGO NOE Project, European Framework Program 7, http://fp7-flamingo.eu 